\title{
Microstructure of Shear Localization in Low Carbon Ferrite-Pearlite Steel
}

\author{
Y. B. XU and Z. G. WANG \\ Institute of Mechanics, Academia Sinica, Shenyang (China) \\ X. L. HUANG, D. XING and Y. L. BAI \\ Institute of Mechanics, Academia Sinica, Shenyang (China) \\ (Received June 30, 1988; in revised form January 23, 1989)
}

\begin{abstract}
A study has been made of the microstructure of the thermally assisted band in a low carbon ferritepearlite steel, resulting from high speed torsional testing with an average strain rate of about 1500 $s^{-1}$. Metallographic examination showed that there are several fine shear bands distributed over a deformed region (the gauge length of the specimen). The width of these bands is estimated to be of the order of magnitude of $50 \mu \mathrm{m}$, and the spacing between them is roughly about $100 \mu \mathrm{m}$. Detailed scanning electron microscopy studies indicate that damage of the microstructure within the band is very apparent, as evidenced by microcrack initiation and coalescence along the shear deformation band. However, there is no evidence that the material in the band had become microcrystalline or non-crystalline.

Thin foil observations using transmission electron microscopy reveal that the density of dislocations within the band is extremely high and the tangled arrangement of dislocations tends to align along the length of the shear band. The grains in the band were also elongated along the shear band and clearly exhibited a crystallographic nature.
\end{abstract}

\section{Introduction}

The thermally assisted shear localization phenomenon which occurs during a dynamic deformation process is a common and important mode of deformation in many metals and alloys when they are deformed to a large plastic strain at high strain rates. It acts as sites for the initiation and growth of microcracks and can lead to catastrophic low ductility or low toughness failure.

Studies on the formation mechanism of the thermally assisted band can help us to understand further the dynamic mechanical behaviour of materials.

Thermally assisted shear localization of structural materials has been the subject of experimental and theoretical investigations in the past [1-4]. A variety of plasticity models have been proposed to describe the shear localization phenomenon [5-7] but the relationship between the mechanical conditions for the formation of a macroscopic shear band and the features on a microstructural level is not well understood at present.

In this paper, we present the results of studies on the microstructural features of the shear localization which occurs at high speed torsional testing in a low carbon ferrite-pearlite steel.

\section{Materials and procedures}

The low carbon steel used for the present study was made in an open-hearth furnace. The ingots were press forged into steel bars. The specimens for the torsional testing were machined from the hot-rolled bar annealed at $900{ }^{\circ} \mathrm{C}$. The chemical composition of the steel is as follows: $0.22 \mathrm{wt} . \% \mathrm{C}, 0.009 \mathrm{wt} . \% \mathrm{~S}, 0.35 \mathrm{wt} . \%$ $\mathrm{Si}, 0.53 \mathrm{wt} . \% \mathrm{Mn}, 0.018 \mathrm{wt} . \% \mathrm{P}$ and balance iron.

Figure 1 shows the profile and dimensions of the specimen which is a short thin-walled tube with $2.0 \mathrm{~mm}$ gauge length, $10.0 \mathrm{~mm}$ internal diameter and about $0.5 \mathrm{~mm}$ wall thickness. Figure 2 shows the macroscopic section of the specimen for structural examinations.

The torsional test was conducted on a torsional split Hopkinson bar. The average strain rate was about $1500 \mathrm{~s}^{-1}$. Specimens for both optical and scanning electron microscopy examinations were sectioned after torsional testing 


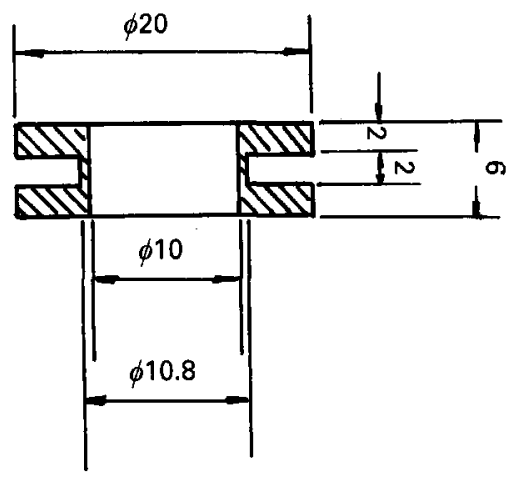

Fig. 1. The profile and dimensions (in millimetres) of the specimen.

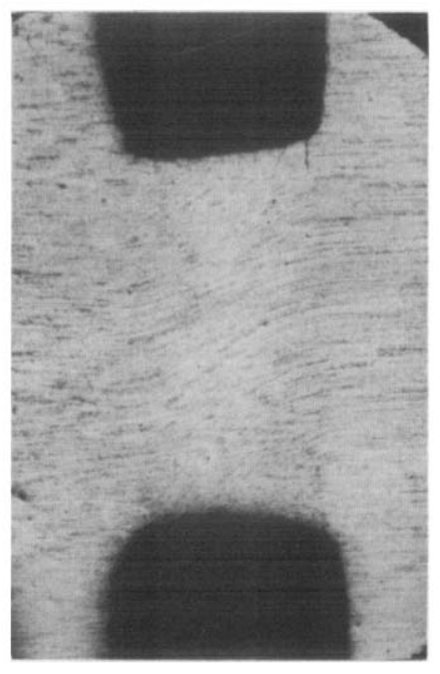

Fig. 2. Macroscopic section of the specimen.

by spark machining and prepared by standard metallographic grinding, polishing and etching methods.

Observations by transmission electron microscopy were performed on a Philips 42 electron microscope operated at $100 \mathrm{kV}$, including both electron diffraction and thin foil techniques. The thin foils for transmission electron microscopy were prepared using a twin-jet electropolisher equipped with a cooling bath. The electropolishing solution consisted of $10 \%$ perchloric acid and $90 \%$ alcohol. The voltage used was $100 \mathrm{kV}$, and the current $75 \mathrm{~A}$. The temperature was $-40{ }^{\circ} \mathrm{C}$.

\section{Results and discussion}

\subsection{Mechanical behaviour}

A typical shear stress-strain curve is shown in Fig. 3, from which it is clear that the work-

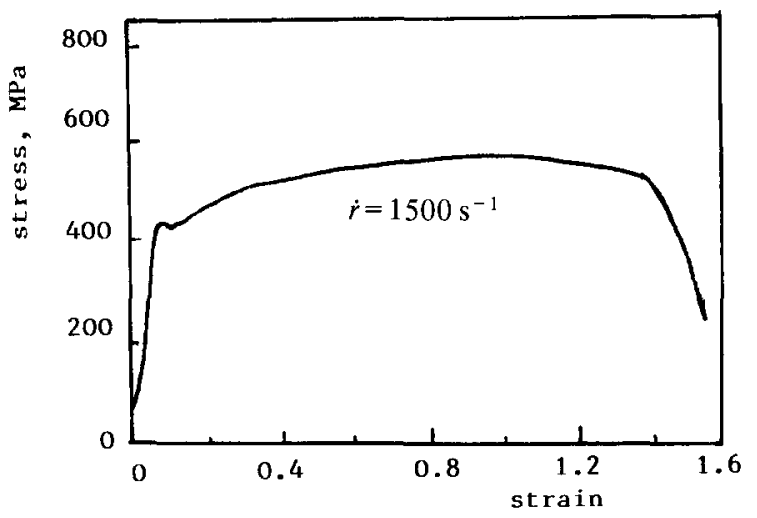

Fig. 3. The stress-strain curve of the test specimen.

hardening rate change from positive to negative at a critical strain corresponded to the presence of the shear deformation bands in this material. The dynamic recording and measurement of deformed specimens show good agreement with the critical strain, ranging from 0.8 to 0.9 [8]. It is therefore concluded that the localized shear does indeed occur at a high strain rate in the low carbon ferrite-pearlite steel, which is in good agreement with the results obtained by Hartley et al. [9]. Their results show that the shear instability occurred at a shear strain between 0.55 and 1.40 in 1020 hot-rolled steel, which is similar to the results of Costin and Duffy [10].

Microhardness measurements at room temperature across the shear band show the typical extreme hardness of such a band. On both sides of the band are regions of deformed material that have suffered various degrees of deformation strengthening and structural softening.

The microhardness value in the deformed region decreases with increasing distance from the band centre until the matrix hardness is reached, as shown in Fig. 4. The microhardness values in ferrite and pearlite structures were determined to be about 200 and 280 respectively.

It should be pointed out that the variation in hardness value in the band is very apparent. The maximum value reached is 400 , and the minimum value is almost as low as that of ferrite. The reason for this is attributed mainly to the severe structural damage in pearlite in the bands.

\subsection{Microstructure in the shear band}

A typical microstructure pattern, including both the deformed region (the gauge length of specimen) and the undeformed regions (steel matrix), is shown in Fig. 5, where the micro- 
structure is composed of both ferrite and pearlite. In the undeformed region, the structure is typical equiaxed whereas, in the deformed region, either ferrite grains or pearlite colonies were rotated and elongated to some extent along the direction of shear band. Careful observations showed that several fine shear bands are distributed over the deformed region as shown in Fig. 6 by arrows. This finding in the present experimental case has seldom been reported in the literature. We con-

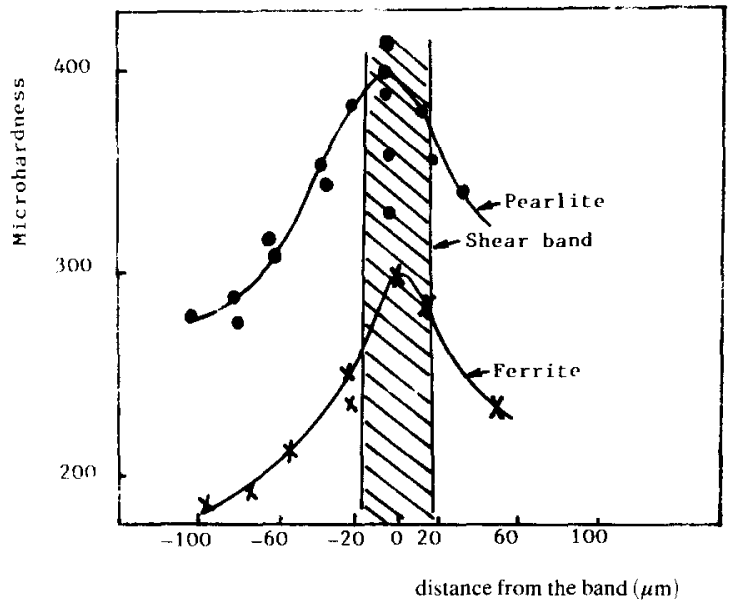

Fig. 4. Microhardness in both the pearlite and the ferrite structures. sider that this is not a single unloading effect but is related to both the thermal effect and the microstructure.

A series of scanning electron microscopy observations revealed that the microcrack initiation in the band occurred by three different distinct processes: decohesion at the ferrite grain-pearlite colony interfaces, decohesion at the ferrite grain boundaries and cracking at

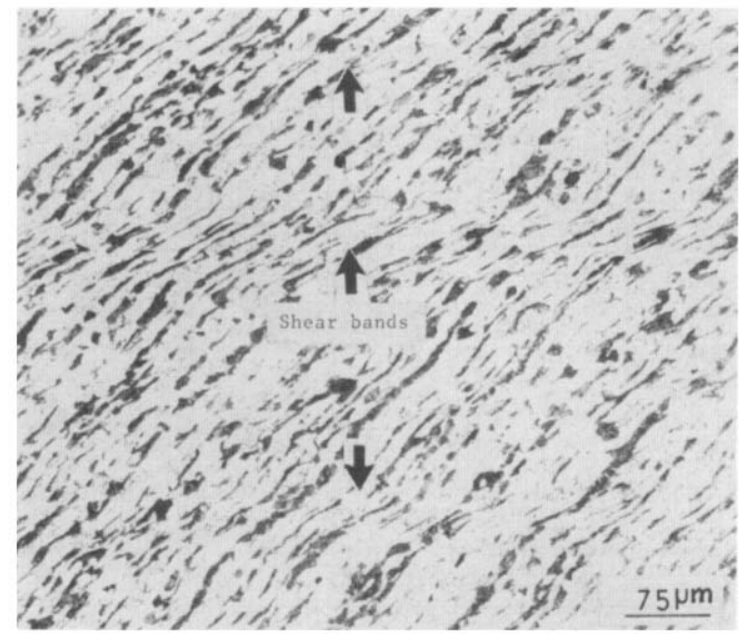

Fig. 6. Several fine shear bands which were found to be distributed over the deformed region (indicated by arrows).

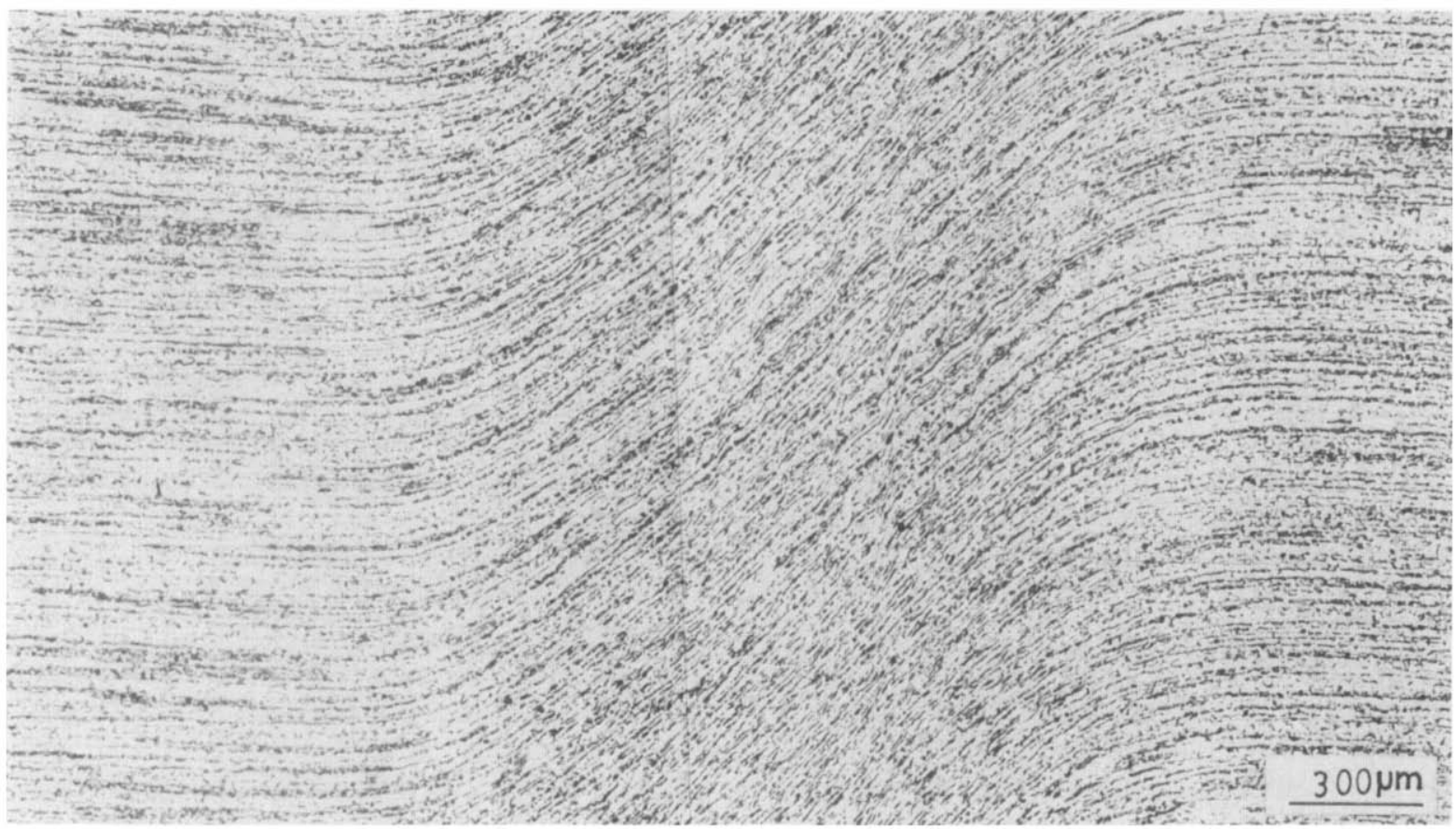

Fig. 5. The localized shear in the low carbon hot-rolled ferrite-pearlite steel. 


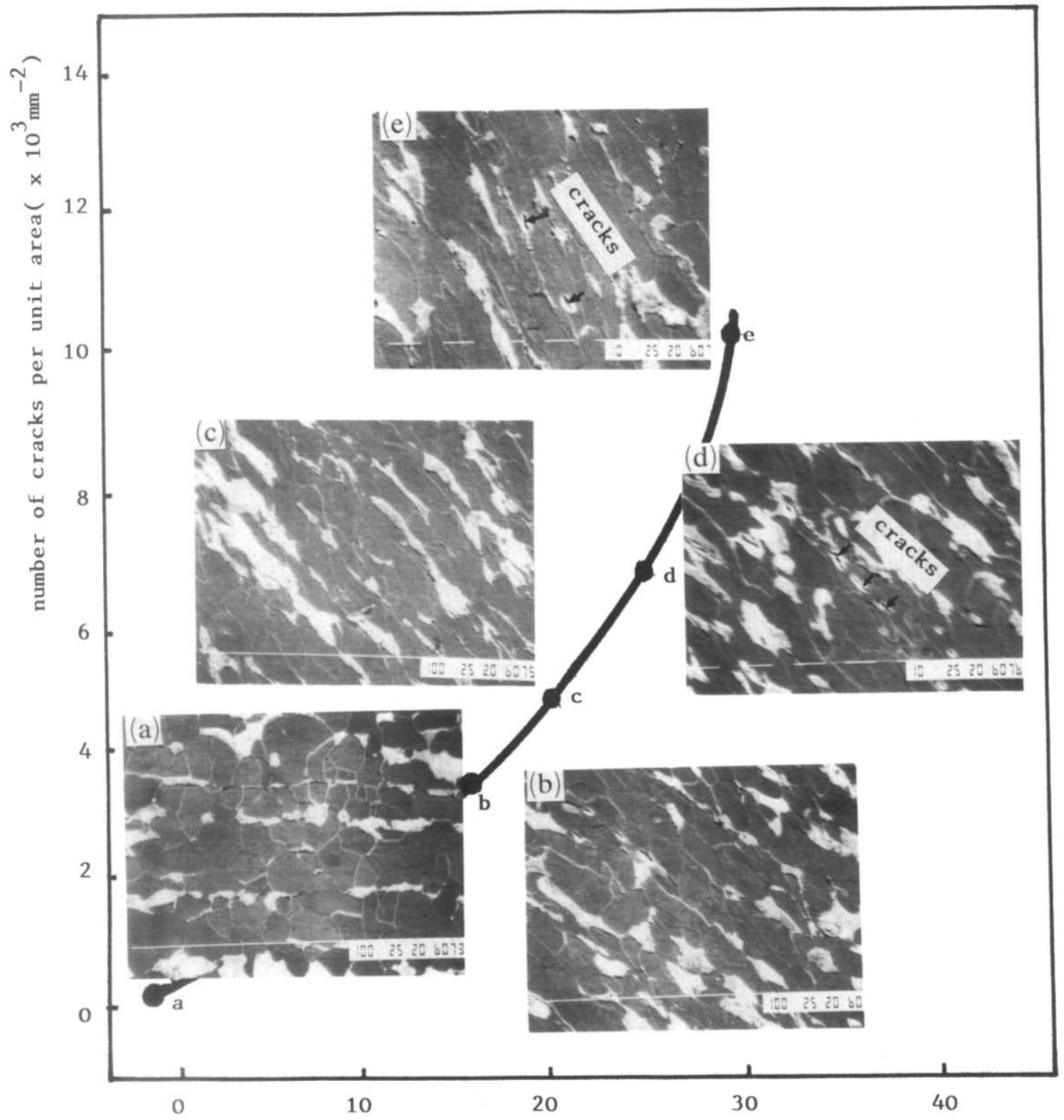

Fig. 7. Diagram showing the relationship between the number of cracks per square millimetre and the distance from the interface between deformed and undeformed regions.

cementite lamellae (Fig. 7) [8]. It is obvious that the structural damage in the band is very severe. The degree of structural damage in the band leading to final failure can be assessed by scanning electron microscopy as shown in Fig. 7. The number of cracks per unit area (which is an indication of the degree of structural damage), was determined as a function of the distance which corresponds to the strain at the interface between the deformed and undeformed regions. It is clear that, the larger the deformation, the more severe will be the degree of structural damage.

A typical microscopic shear band which crossed several grains observed by transmission electron microscopy is shown in Fig. 8. The thin foil in this case is approximately perpendicular to the final fracture surface. It is obvious that the grains within the band were elongated along the band and that the density of dislocations in the shear band is extremely high (Fig. 9) in comparison with that outside the band; the tangled 


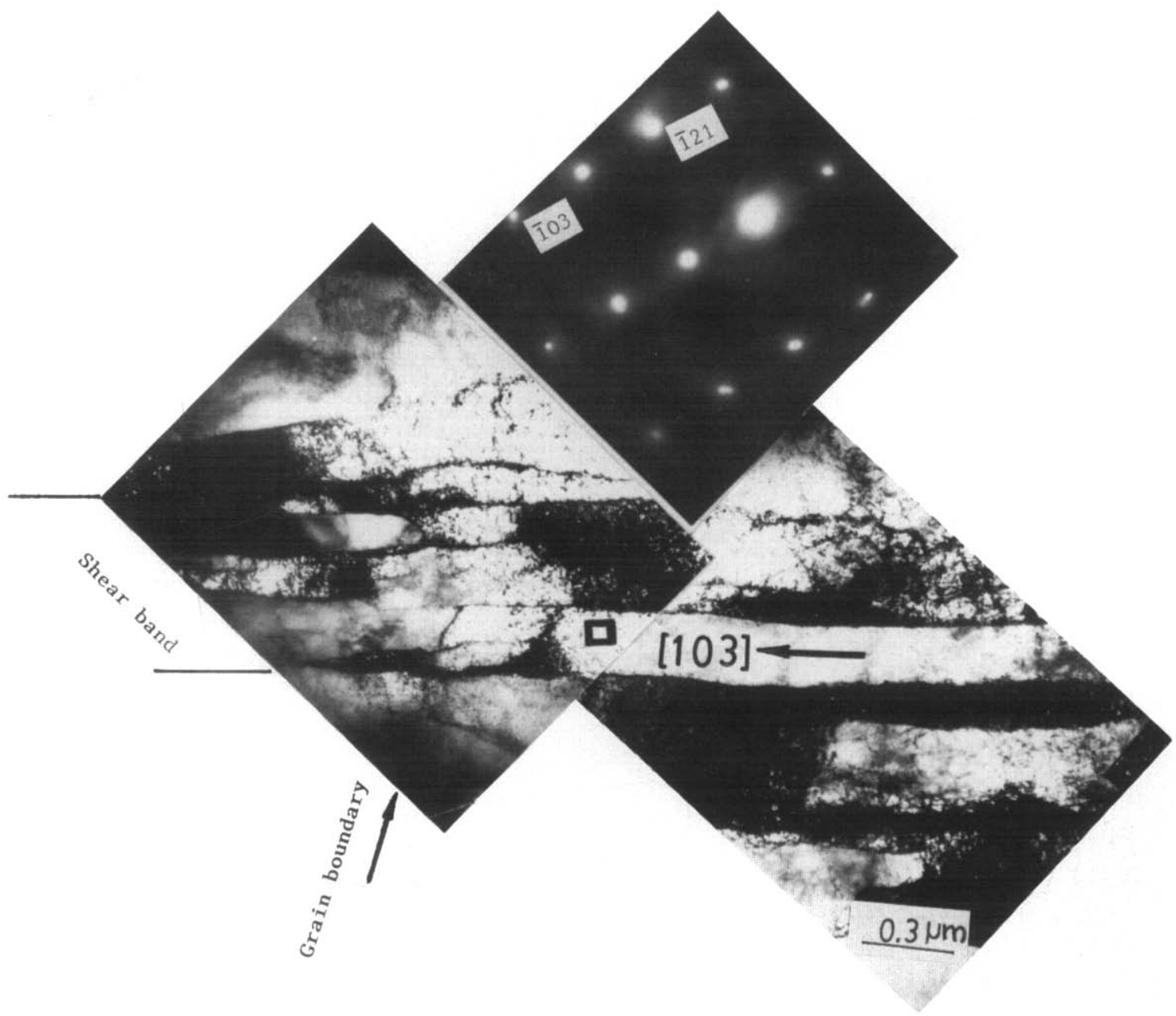

Fig. 8. Transmission electron micrograph showing the structure and crystallographic nature in the microscopic shear band.

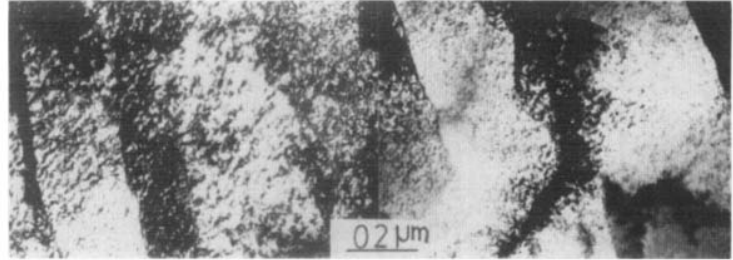

Fig. 9. The heavy density of dislocations in the shear band.

arrangement of dislocations tends to align along the length of the shear band (Fig. 10). Electron diffraction analysis reveals that the shear band in Fig. 8 has a clear crystallographic relation in which the trace direction is [103]. This situation can be seen more clearly in Fig. 11 where the

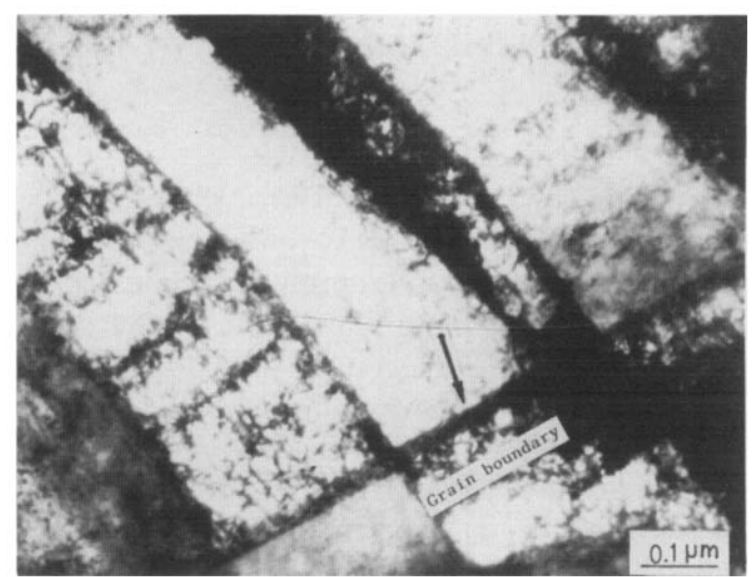

Fig. 10. The tangled arrangement of dislocations which tends to align along the length of the shear band. 


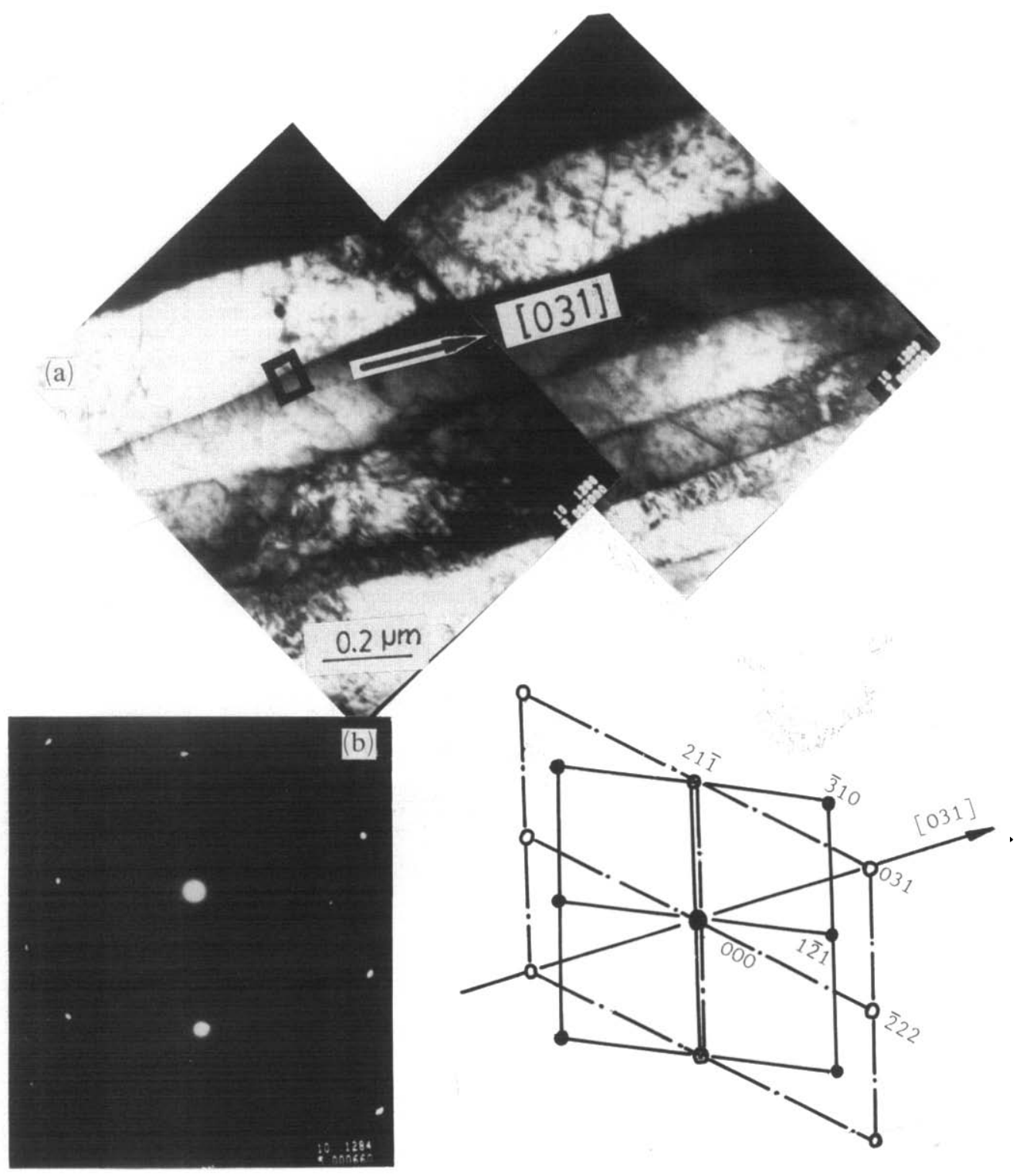

Fig. 11. Microstructural feature of the localized shear deformation band: (a) bright field micrograph showing a long and straight shear band; (b) selected-area diffraction pattern from the square area in $(a) ;(c)$ indexing of the pattern $(\bullet[135] ; 0,[213])$ $\left([2 \mathrm{i} 3]_{\alpha_{1}} / /[135]_{\alpha_{2}}\right)$.

electron diffraction pattern was taken from the interface between the two grains as indicated by the square in the band. The trace of the shear band is in agreement with the direction [013] of the intersection of the foil plane [213). The tangled arrangement of dislocations and the heavy density of dislocations in the shear band suggest that the material in the band suffered a heavy deformation and highly localized shear but had not yet become microcrystalline or noncrystalline in this case.
From both the observations and the analysis mentioned above, it can be assumed that the microscopic shear band first occurs as a result of the crystallographic slip on a dominant slip system in one grain and then propagates into adjacent grains by multiple- or cross-slip events because of stress concentration at the grain boundaries. The general observations show that the origin of the initial localized shear event resulting in the formation of the shear band prior to the final fracture is related to the structural 
softening or damage. The present work shows that the large strain in the shear band must be responsible for microcrack initiation and coalescence as well as final fracture along the localized shear band.

\section{Conclusions}

The current result indicate the following.

(1) Several fine shear bands have been found to distribute uniformly over the gauge length of specimen in the low carbon hot-rolled ferrite-pearlite steel.

(2) The heavy density of dislocations and the tangled arrangement of dislocations suggest that the material in the band has suffered heavy deformation and localized shear but has not become microcrystalline or non-crystalline.

(3) The microstructural damage in the shear band is extremely severe, as evidenced by crack initiation and coalescence, and the final fracture occurred along the localized shear band.

\section{Acknowledgment}

This work was supported by the Chinese Academy of Sciences under Special Grant 87-52.

\section{References}

1 H. C. Rogers, Annu. Rev. Mater. Sci., 9 (1979) 238.

2 S. P. Timothy and I. M. Mutchings, Met. Sci. Technol, I (1985) 526 .

3 H. A. Grebe, H. R. Pak and M. A. Meyers, Metall. Trans. A, $16(1985) 761$.

4 D. A. Shockey, in L. E. Murr, K. T. Staudhammer and M. A. Meyers, Proc. Conf. on Metallurgical Applications of Shock-Wave and High Strain-rate Phenomena, Marcel Dekker, New York, 1986, p. 1.

5 Y. L. Bai, J. Mech. Phys. Solids, 30(1982) 195.

6 E. Rauch, G. R. Canova, J. J. Jonas and S. L. Semiatin, Acta Metall., 33 (1985) 465.

7 A. M. Merzer, J. Mech. Phys. Solids, 30(1982) 5.

8 X. L. Huang, Masters Degree Thesis. Institute of Mechanics, Academia Sinica, 1987.

9 K. A. Hartley, J. Duffy and R. H. Hawley, J. Mech. Phys. Solids, 35 (1987) 283.

10 L. S. Costin and J. Duffy, J. Eng. Mater., I0/ (1979) 258. 